\title{
Pathological Features of Pancreatic Endocrine Tumor and Its Differential Diagnosis with Acinic Cell Carcinoma
}

\author{
Zichuang Wang, Xiaoyong Chen \\ Henan University of Traditional Chinese Medicine, Zhengzhou Henan, 450002, China
}

\begin{abstract}
Keywords: Pancreatic endocrine tumor, Pancreatic acinar cell carcinoma, Pathological features, Differential diagnosis
\end{abstract}

\begin{abstract}
Purpose: analyze the pathological features of pancreatic endocrine tumor and discuss the differential diagnosis of pancreatic endocrine tumor and pancreatic acinic cell carcinoma. Method: choose 10 patients of pancreatic endocrine tumor from the Chinese Medicine Hospital of Henan Province and the Tumor Consultation Center of Henan Province during January 2012 to January 2015 as research object to analyze their pathological features; choose another 10 patients of pancreatic acinic cell carcinoma conformed during the same period to make control study, combine the pathological examination and documentations to male differential diagnosis for pancreatic endocrine tumor and pancreatic acinic cell carcinoma. Result: (1)for cell morphology, tumor cells of the pancreatic endocrine tumor are arranged as solid flakes, aciniform and trabecular, its organizational structure is similar with that of pancreatic acinic cell carcinoma, but the organization of pancreatic endocrine tumor has no heterocyst, while the organization of pancreatic acinic cell carcinoma has heterocyst. (2) For the expression of immunohistochemistry, the pan cytokeratin, synaptophysin and chromogranin of the patients of pancreatic endocrine tumor and pancreatic acinic cell carcinoma are all positive, while the expression of vimentin, epithelial membrane antigen and insulin of the two kinds of pancreatic tumor are different. (3) For Clinical biological behavior, only one of the ten pancreatic endocrine tumor was diagnosed to be pancreatic tail neuroendocrine carcinoma, tumor of other nine patients were benign, while ten pancreatic acinar cell carcinoma were all high aggressive malignant tumor. Conclusion: Most of the pancreatic endocrine tumors are benign, while pancreatic acinar cell carcinoma is a kind of malignant tumor, though the cellular morphology of two kinds of tumor are similar, while there is obvious difference on the aspect of biological behavior of two kinds of tumors, clinical differential diagnosis of these two kinds of tumors should be paid attention.
\end{abstract}

\section{Introduction}

Pancreas is an important gland of human's body, it is an important secretory organ. Pancreatic endocrine tumor is a kind of tumor grows in the pancreas, mainly caused by the abnormal pancreatic endocrine. The cellular morphology of pancreatic endocrine tumor is pretty similar with that of pancreatic acinar cell carcinoma, these two kinds of pancreatic tumors are easy to be confused clinically, which is extremely unfavorable for the clinic treatment of tumors. Therefore, it is necessary to make further research on the differential diagnosis of pancreatic endocrine tumor and pancreatic acinar cell carcinoma. According to relevant research reports, it is hard to distinguish pancreatic endocrine tumor and pancreatic acinar cell carcinoma purely depends on cellular morphology, clinical diagnosis should combine clinical applications, cellular morphology and immunohistochemical analysis ${ }^{[1]}$. This research chose 10 pancreatic endocrine tumor patients and 10 pancreatic acinar cell carcinoma patients to make comparison, selected from the Chinese Medicine Hospital of Henan Province and the Tumor Consultation Center of Henan Province during January 2012 to January 2015, based on the cellular morphology test and immunohistochemical staining of patients to analyze the pathological features of pancreatic endocrine tumor and discuss the differential diagnosis of pancreatic endocrine tumor and pancreatic acinar cell carcinoma. Now sort analysis as follows: 


\section{Clinical data and research method}

Clinical data. Choose 10 patients of pancreatic endocrine tumor from the Chinese Medicine Hospital of Henan Province and the Tumor Consultation Center of Henan Province during January 2012 to January 2015 as research object. All patients were diagnosed to be pancreatic endocrine tumor, 5 male patients and 5 female patient at the age of 35 61 years old, average (51.67 \pm 8.37 )years old. All the 10 patients had the symptom of repetitious chest distress, cold sweat and palpitation, dull pain of the upper abdomen of one patient lasted for half an year and spread to the shoulder-back. All patients had the excision of pancreatic tail and were found lumps in different sizes in the pancreatic tail. Intake this 10 patients into this research based on the agreements of their families and themselves.

Choose another 10 patients of pancreatic acinic cell carcinoma conformed during the same period to make control study, all patients were diagnosed to be pancreatic acinic cell carcinoma, 6 male patients and 4 female patient at the age of 36 67 years old, average (52.37 \pm 8.56$)$ years old. Intake this 10 patients into this research based on the agreements of their families and themselves.

Make statistical comparative analysis for the two groups of patients based on their clinical data, $\mathrm{P}>0.05$, can make research comparison.

Research method. Analyze the clinical data of the 10 pancreatic endocrine tumor patients, collect the tissue dissection of pancreatic endocrine tumor in surgery, fix the tissue samples with neutral formalin at concentration of $10 \%$ after the surgery, paraffin-embedded, cut it into section preparation with thickness of $5 \mu \mathrm{m}$, HE staining, make cell morphology test. Use the antibody produced by Dako company, use immunohistochimie two anti - enzyme mark polymer (Envision) method to make immunohistochemical staining to analyze its pathological features.

Detailed procedures of the immunohistochimie two anti - enzyme mark polymer (Envision) method are as follows: xylene conventional dewaxing process, twice, 10 minutes per time; use gradient ethanol to hydrate, soak in turn according to the order of $100 \%, 90 \%, 80 \%, 70 \%$, each soak 1 minute. wash with distilled water, washing time refers to the specific situation; make endogenous enzymes removing with hydrogen peroxide at concentration of 3\%, place for 10 minutes under the room temperature; consecutively wash twice with distilled water, 3 minutes per time; put the section into citrate buffer, and place it into the microwave oven, place it under the room temperature 15 minutes after boiling for natural cooling; consecutively wash twice with distilled water, 3 minutes per time; consecutively wash twice in phosphate buffered saline(PBS), 3 minutes per time; set the temperature as $37^{\circ} \mathrm{C}$, cultivate for 30 minutes in the PV-9000Po1v-HRPanti-Mouse/RabbitIgG kit; consecutively wash twice in phosphate buffered saline(PBS), 3 minutes per time; make color processing with $\mathrm{DAB}$ color developing agent, observe the dyeing of cell membrane under the microscope; after observation, wash with tap water for 5 minutes; use hematoxylin to make redyeing, then wash with water; make color separation for $2 \mathrm{~s}$ with acidic alcohol at concentration of $1 \%$, then wash; use gradient ethanol to hydrate, soak in turn according to the order of $70 \%, 80 \%, 90 \%, 100 \%$, each soak 2 minute; Place the section under room temperature for natural withering, after drying, make sealing pieces processing with neutral balsam.

Search relevant documentations through key word like "pancreatic acinar cell carcinoma”, "cell morphology”, "immunohistochemistry", "biological behavior" in the database of HowNet, Wanfang and Vip, combine the pathological examination of 10 pancreatic acinar cell carcinoma patients with documentations to analyze its pathological features, make differential diagnosis for the histopathological characteristics and clinical biological behavior of pancreatic endocrine tumor and pancreatic acinar cell carcinoma.

Statistical method. Log data in the SPSS17.0 software and process it. The enumeration data is expressed as $[\mathrm{n}(\%)]$, make $x^{2}$ inspection; when the enumeration data is expressed as $(\bar{x} \pm s)$ make $t$ inspection. Use $\mathrm{P}<0.05$ to express that the data difference has statistical significance. 


\section{Result}

Cell morphology. For cell morphology, tumor cells of the pancreatic endocrine tumor are arranged as solid flakes, partly aciniform and trabecular, its organizational structure is similar with that of pancreatic acinic cell carcinoma, but the organization of pancreatic endocrine tumor has no heterocyst, there are abundant blood vessels in the intercellular substance, and no obvious necrocytosis; while the organization of pancreatic acinic cell carcinoma has heterocyst and has obvious necrocytosis and blood vessels are not abundant.

Expression immunohistochemistry. For the expression of immunohistochemistry, the pan cytokeratin, synaptophysin and chromogranin of the patients of pancreatic endocrine tumor and pancreatic acinic cell carcinoma are all positive, Ki-67 nuclear antigen is less than $2 \%$; while the expression of vimentin, epithelial membrane antigen and insulin of pancreatic endocrine tumor patients are positive, that of the pancreatic acinic cell carcinoma patients are negative.

Clinical biological behavior. For clinical biological behavior, five of the ten pancreatic endocrine tumor patients were diagnosed to be pancreatic tail neuroendocrine carcinoma; four of them were diagnosed to be high aggressive malignant tumor, its clinical biological behavior cannot be confirmed; one was diagnosed to be pancreatic tail neuroendocrine carcinoma, has no differentiation, mitotic figure has about 10 high power field, there are 14 lymphatic gland surrounding the pancreas, all have no transition. Nine patients of the pancreatic endocrine tumor were benign, while ten pancreatic acinar cell carcinoma were all high aggressive malignant tumor.

\section{Conclusion}

Pancreatic endocrine tumor, also called as "pancreatic tumor" is a kind of tumor mainly caused by the abnormal insulin of the pancreatic endocrine. At present, affection mechanism of the pancreatic endocrine tumor is not clear on clinic. Some scholars believe that pancreatic endocrine tumor is mainly originated from duct pancreatic stem cells, duct pancreatic stem cells can have large number of breeding, and has multilineage differentiation, under the catalysis of carcinogen, it will differentiate into islet cell, acinous cell and ductal cell, after the pancreatic stem cells differentiation, the gene will change, the ability of cell proliferation and differentiation has lost control, large number of uncontrollable breeding and differentiation will appear and form pancreatic tumor ${ }^{[2]}$. The basis of this hypothesis is mainly the new islet cell that invisible in the duct of pancreatic endocrine tumor, while it is not certificated, the onset mechanism of pancreatic endocrine tumor is still in dispute.

Pancreatic endocrine tumor mainly appears in the pancreatic tail, this mainly because pancreatic stem cells concentrate in the pancreatic cell. In 1999, World Health Organization divided pancreatic endocrine tumor into non-functionality and functionality, hormone level of these two kinds are improved obviously, their difference lies in whether there is hormone syndrome. Pancreatic endocrine tumor is a kind of rare pancreatic tumor, it morbidity only accounts for $1 \%$ of pancreatic tumor, and mainly incidents in the aged population ${ }^{[3]}$. The clinical diagnosis of pancreatic endocrine tumor is mainly by observing cell morphology, but many clinical researches showed that pure cell morphology observation cannot make effective differential diagnosis for the pancreatic endocrine tumor, it is easy to be confused with pancreatic acinar cell carcinoma, thus may lead to misdiagnose and delay the treatment opportunity. Therefore, the further research on the differential diagnosis of pancreatic endocrine tumor and pancreatic acinar cell carcinoma is necessary, has an important significance.

This research chose 10 pancreatic endocrine tumor patients and 10 pancreatic acinic cell carcinoma patients for comparative study, after analyzing the pathological feature of pancreatic endocrine tumor we found that for cell morphology, tumor cells of the pancreatic endocrine tumor are arranged as solid flakes, partly aciniform and trabecular, its organizational structure is similar with that of pancreatic acinic cell carcinoma, but the organization of pancreatic endocrine tumor has no heterocyst, there are abundant blood vessels in the intercellular substance, and no obvious necrocytosis;for the expression of immunohistochemistry, the pan cytokeratin, synaptophysin and chromogranin of the patients of pancreatic endocrine tumor and pancreatic acinic cell carcinoma are 
all positive, while the expression of vimentin, epithelial membrane antigen and insulin of the two kinds of pancreatic tumor are different. Pan cytokeratin, synaptophysin, chromogranin and insulin are all expression sign of pancreatic endocrine tumor, normally pan cytokeratin, synaptophysin and chromogranin are expressed as positive, while as expression sign of hormone specificity, insulin can effectively reflects pancreatic hormone levels, its expression will be different from cancerization degree.

In this research, compare pancreatic endocrine tumor and pancreatic acinic cell carcinoma to discuss the differential diagnosis of two kinds of pancreatic tumors. The biological behavior difference of two kinds of pancreatic tumors is mainly expressed in the atypia, lymphatic metastasis and tumor characteristics ${ }^{[5]}$ of cell, though cell morphology of these two kinds of pancreatic tumors are similar, but the organization of pancreatic endocrine tumor has no heterocyst, while the organization of pancreatic acinic cell carcinoma has heterocyst;Lymph gland of pancreatic endocrine tumor has no transition, while the pancreatic acinar cell carcinoma has diffuse transition; only one of the ten pancreatic endocrine tumor was diagnosed to be pancreatic tail neuroendocrine carcinoma, while ten pancreatic acinar cell carcinoma were all high aggressive malignant tumor.

In conclusion, most of pancreatic endocrine tumor are benign, while pancreatic acinar cell carcinoma is a kind of malignant tumor, though with similar cell morphology, their biological behaviors are obvious different, differential diagnosis of these two kinds of tumors should be paid special attention.

\section{References}

[1] Sikkens E C M ,Cahen D L ,De Wit J, etal. A prospective assessment of the natural course of the exocrine pancreatic function in patients with a pancreatic head tumor.Journal of clinical gastroenterology,2014,48(5):e43-e46.

[2] Pan Yulin, Xie Yun, Luo Zhimou and so on. Pathological Diagnosis and Differential Diagnosis of Pancreatic Endocrine Tumor and Pancreatic Acinar Cell Carcinoma .Journal of Bengbu Medical College, 2013,38(4):421-423,426.

[3] Yang Lijuan. Analysis on the Pathological Diagnosis and Differential Diagnosis of Pancreatic Endocrine Tumor and Pancreatic Acinar Cell Carcinoma. China Practical Medicine, 2014,9(22):66-67.

[4] Burns W R ,Edil B H .Neuroendocrine pancreatic tumors: Guidelines for management and update.Current treatment options in oncology,2012,13(1):24-34.

[5] McCall C M ,Shi C ,Cornish T C, etal. Grading of well-differentiated pancreatic Neuroendocrine tumors is improved by the inclusion of both ki67 Proliferative index and mitotic rate.American Journal of Surgical Pathology,2013,37(11):1671-1677. 